\title{
Role of phosphatidic acid in plant galactolipid synthesis
}

\author{
Emmanuelle Dubots ${ }^{1}$, Cyrille Botté, Laurence Boudière, Yoshiki Yamaryo-Botté, \\ Juliette Jouhet, Eric Maréchal, Maryse A. Block*
}

Laboratoire de Physiologie Cellulaire et Végétale, UMR 5168 CNRS, CEA, INRA, Université Joseph Fourier Grenoble I, Institut de Recherches en Sciences et Technologies pour le Vivant, CEA-Grenoble, 17 rue des Martyrs, 38054 Grenoble, France

\begin{abstract}
Phosphatidic acid (PA) is a precursor metabolite for phosphoglycerolipids and also for galactoglycerolipids, which are essential lipids for formation of plant membranes. PA has in addition a main regulatory role in a number of developmental processes notably in the response of the plant to environmental stresses. We review here the different pools of PA dispatched at different locations in the plant cell and how these pools are modified in different growth conditions, particularly during plastid membrane biogenesis and when the plant is exposed to phosphate deprivation. We analyze how these modifications can affect galactolipid synthesis by tuning the activity of MGD1 enzyme allowing a coupling of phosphoand galactolipid metabolisms. Some mechanisms are considered to explain how physicochemical properties of PA allow this lipid to act as a central internal sensor in plant physiology.
\end{abstract}

\section{Introduction}

In plants, phosphatidic acid (PA) is both a metabolic precursor for all glycerolipids and a key signalling lipid that regulates numerous reactions involved in developmental and physiological processes particularly in response to environmental stresses. Several pools of PA are involved, associated with different cell membranes. Some of these sources of PA represent potential precursor supplies for synthesis of galactoglycerolipids, which are the main glycerolipids of plants and the focus of the present review.

One of the specificities of the plant cells is indeed their high content in galactolipids. In plastids, a family of plant specific organelles including the chloroplast, MGDG (monogalactosyldiacylglycerol) and DGDG (digalactosyldiacylglycerol) represent together close to $80 \%$ of membrane lipids. In chloroplasts, MGDG and DGDG are essential for photosynthesis. Galactolipids are mainly restricted to plastid membranes, however, when the plant grows under Pi restriction, DGDG can replace phospholipids in some non-plastid membranes such as the tonoplast, the plasma

\footnotetext{
* Corresponding author. CEA-Grenoble, iRTSV/LPCV, 17 Rue des Martyrs, 38054 Grenoble, France. Tel.: +33 (0) 4387849 85; fax: +33 (0) 438785091.

E-mail address: mblock@cea.fr (M.A. Block).

1 Present address: Biochemistry Unit, Department of Biology, University of Fribourg, $\mathrm{CH}-1700$ Fribourg, Switzerland.
}

membrane and the mitochondrial membrane and thus represents a major lipid at the whole cell level. This spectacular lipid remodelling represents a way to save Pi for other cell functions. The role of galactolipids for cellular membrane remodelling under Pi deprivation is another indication of the vital importance of galactolipids for plants.

Galactolipid formation is initiated by the transfer of a galactose from UDP-galactose onto diacylglycerol. This step is catalyzed by MGDG synthases (which in Arabidopsis are AtMGD1, AMGD2, AtMGD3) located in the plastid envelope [1]. Diacylglycerol supplied to MGDG synthases results from hydrolysis of PA and recent investigations indicate that in the chloroplast envelope MGD1 is activated by PA [2]. In the chloroplast envelope, PA is also the precursor for the synthesis of phosphatidylglycerol, PG, a phospholipid, which is another essential component of the chloroplast membranes [3]. In this context it is important to understand and connect the distinct roles of PA as an intermediate metabolite in formation of the plant cell membranes and as a mediator in the transduction of signal in a series of physiological contexts. We will here inventory the different steps where PA is involved in the galactolipid synthetic pathway. We will focus on the photosynthetic plant cell and give a brief report on some recently investigated parasites of medical importance, i.e. the Apicomplexans, which happen to harbour a non-photosynthetic plastid, the apicoplast [4-6]. We will then consider how PA can connect galactolipid synthesis with basic functions of the plant cell. 


\section{Levels of phosphatidic acid in plant cells}

In leaves, PA generally represents $1-5 \mathrm{~mol} \%$ of total glycerolipids but in some stress conditions the concentration can highly increase [7]. In available reports, PA levels are not always expressed as a mol percentage of total membrane lipids limiting the precision of the comparison, but the magnitude of variations looks nevertheless always important. An increase of PA from 1.2 to $11.5 \mathrm{nmol}$ per mg of dry weight ( 9.6 fold increase) was reported in rosettes of preflowering Arabidopsis plants exposed to freezing [8]. Similarly, an increase of PA from 5 to $25 \mathrm{~mol} \%$ of phospholipids (5 fold increase) was reported in leaves of tomato and Arabidopsis plants treated with high concentration of $\mathrm{NaCl}$ mimicking a drought stress [9] and an increase of PA from 0.5 to $4 \mathrm{~mol} \%$ of total glycerolipids (8 fold increase) was detected in Arabidopsis cells at early steps of phosphate deprivation (Jouhet, personal communication).

Different molecular species of PA are present. They are composed of $\mathrm{C} 16$ and $\mathrm{C} 18$ fatty acids with up to 3 double bonds. The main molecular species are usually $34: 2$ and 34:3 (indicating total number of fatty acyl carbons: total number of double bonds in fatty acids, as often reported in identification obtained by mass spectrometry) but in some stresses highly unsaturated diacyls such as $36: 5$ or $36: 6$ can also appear as the main molecular species $[7,10]$. Lipid analysis of PLD mutants exposed to stresses led to the conclusion that these PA species are diC18 species and derive primarily from PC [8]. In plants exposed to cold stress, detection of
PA enriched in C16:3 [8], a typical fatty acid of MGDG, suggests that some molecular species of PA can derive from MGDG.

$\mathrm{PA}$ is heterogeneously distributed in the different cellular membranes. The plasma membrane is enriched in PA $(3-4 \mathrm{~mol} \%$ of lipids [11]) whereas, in the chloroplast membranes, PA is very low, never detected in the chloroplast envelope, and around $0.1 \mathrm{~mol} \%$ of lipids in tobacco thylakoids as reported by [12]. The authors detected mainly a form of PA with C18:1 at sn-1 and C16:0 at sn-2 position of glycerol, which is considered as a prokaryotic form of glycerolipid typically synthesized in chloroplasts as we will see below.

\section{Phosphatidic acid as an intermediate metabolite in galactolipid synthesis}

PA is produced by a collection of enzymes (see Fig. 1 and Table 1). The neo-synthesis of PA goes through a two step acylation of glycerol-3-P with lyso-PA as an intermediate. These two acylation-steps are more generally the basic way to the de novo formation of phosphoglycerolipids and galactoglycerolipids and PA is therefore an obligate early precursor in synthesis of all glycerolipids. In plants, acylation of glycerol-3-P occurs in three membrane compartments: the ER, the mitochondrial membranes, and the chloroplast envelope. We will not detail here the characteristics of mitochondrial acyltransferases but information can be found in [13]. One point to note is that the mitochondrial

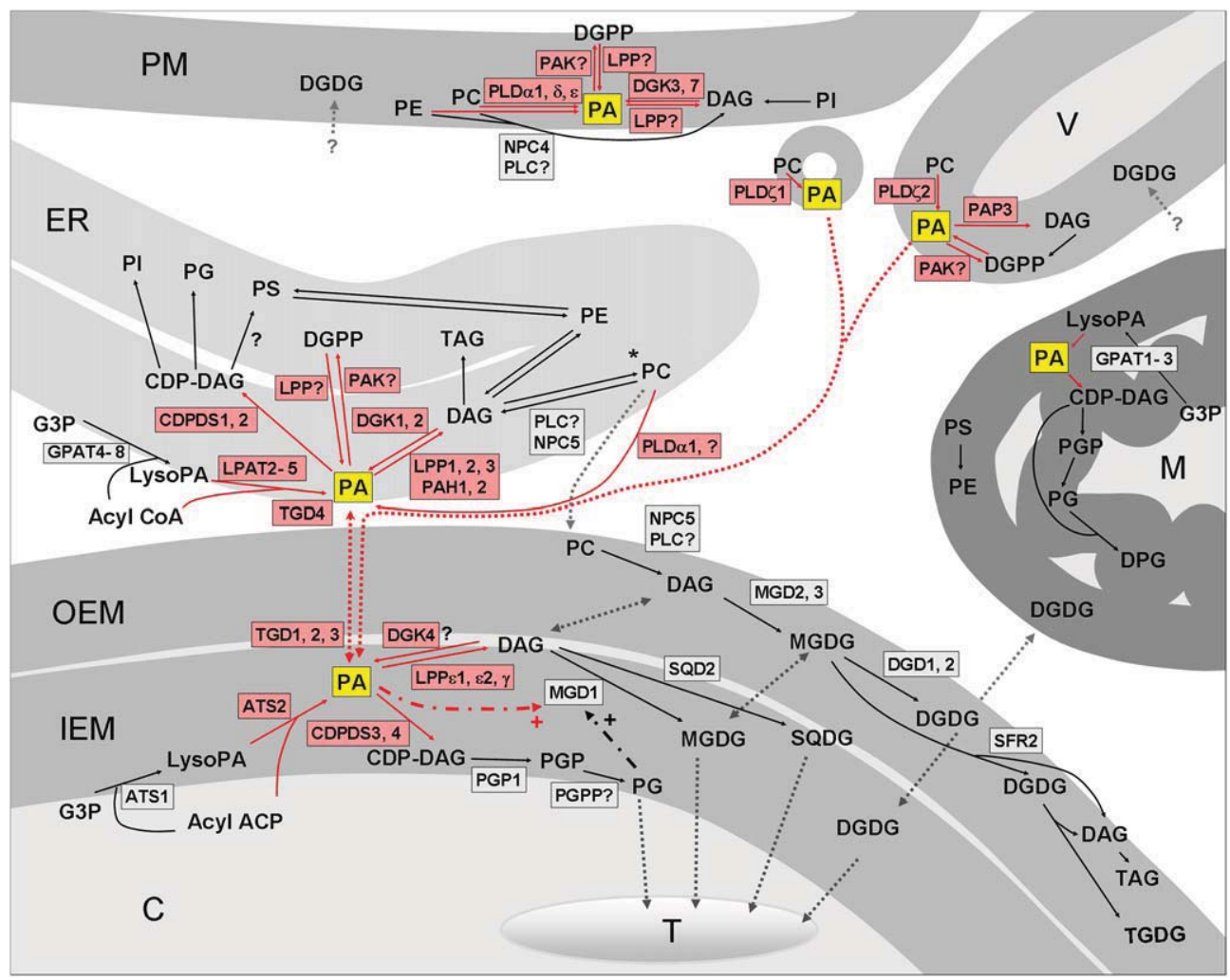

Fig. 1. Origin and fate of the different pools of PA in the plant cell. Several pools of PA are dispatched in the different cell membranes. Different molecular species of PA are present depending of enzyme specificity and substrate availability. For the most part PA is converted to either DAG or CDP-DAG. Balance between the 2 pathways is dependent on the ratio of the activities of the PA phosphatases and the CDP-DAG synthases. CDP-DAG and DAG are substrates in formation of phospholipids and galactolipids [14]. DAG can also generate TAG $([83,84])$. Conversion to DGPP is an alternative although likely less abundant route [85]. Some DAG kinases produce PA possibly even in plastids since some sequences harbour a putative chloroplast transit peptide. PA can modify the activity of some target proteins including several enzymes involved in the lipid synthesis pathways such as the MGDG synthase, MGD1, in the chloroplast envelope and the soluble phosphoethanolamine N-methyltransferase, PEAMT (indicated by * in the scheme), involved in PC synthesis [2,80]. Demonstrations that specific sources of PA are active at another cell location indicate that PA can be transported between membranes (see [2,26]). Enzymes directly related with PA, either producing PA or using PA, are indicated in red. Lipid transports are indicated by a dotted line and activation by a semi-dashed line. Lipid enzymes are listed in Table 1. Abbreviations: PM, plasma membrane; ER, endoplasmic reticulum; V, vacuole; C, chloroplast; T, thylakoids; OEM, outer envelope membrane; IEM, inner envelope membrane. (For interpretation of the references to colour in this figure legend, the reader is referred to the web version of this article.) 
Table 1

Lipid enzymes presented in Fig. 1. Enzyme location refers to [77] when available or to TAIR. For level changes in gene expression under Pi deprivation abbreviations are NR: Not reported, NC: No change, NM: Not in microarray, - or $+: 1.5-2$ fold change range, -- or $++:>2$ fold change range.

\begin{tabular}{|c|c|c|c|c|c|c|}
\hline \multirow[t]{3}{*}{ Name } & \multirow[t]{3}{*}{ Activity } & \multirow[t]{3}{*}{ Location } & \multicolumn{3}{|c|}{ Gene expression under Pi deprivation } & \multirow{3}{*}{$\begin{array}{l}\text { Arabidopsis } \\
\text { locus }\end{array}$} \\
\hline & & & \multicolumn{2}{|c|}{ According to [20] } & \multirow{2}{*}{$\frac{\text { According to [21] }}{\text { Whole seedling }}$} & \\
\hline & & & Leaf & Root & & \\
\hline ATS1 & $\begin{array}{l}\text { Glycerol-3-phosphate } \\
\text { acyltransferase }\end{array}$ & Chloroplast & NR & NR & $\mathrm{NC}$ & At1g32200 \\
\hline ATS2 & Lyso-PA acyltransferase & IEM & NR & NR & -- & At4g30580 \\
\hline CDPDS1 & CDP-DAG synthase & ER & NR & NR & + & At1g62430 \\
\hline CDPDS2 & & & NR & NR & $\mathrm{NC}$ & At4g22340 \\
\hline CDPDS3 & & IEM & NR & NR & $\mathrm{NC}$ & At4g26770 \\
\hline CDPDS4 & & & NR & NR & - & At2g45150 \\
\hline DGD1 & DGDG synthase & OEM & ++ & NR & ++ & At3g11670 \\
\hline DGD2 & & & NR & ++ & ++ & At4g00550 \\
\hline DGK1 & DAG kinase & ER & NR & NR & NC & At5g07920 \\
\hline DGK2 & & & NR & NR & $\mathrm{NC}$ & At5g63770 \\
\hline DGK3 & & PM & NR & NR & + & At2g18730 \\
\hline DGK7 & & & NR & NR & $\mathrm{NC}$ & At4g30340 \\
\hline DGK4 & & Chloroplast? & NR & NR & $\mathrm{NC}$ & At5g57690 \\
\hline GPAT1 & Glycerol-3-phosphate & Mitochondria? & NR & NR & - & At1g06520 \\
\hline GPAT2 & acyltransferase & & ++ & NR & ++ & At1g02390 \\
\hline GPAT3 & & & NR & NR & NC & At4g01950 \\
\hline GPAT4 & & ER? & NR & NR & + & At1g01610 \\
\hline GPAT5 & & & NR & NR & ++ & At3g11430 \\
\hline GPAT6 & & & NR & NR & + & At2g38110 \\
\hline GPAT7 & & & NM & NM & NM & At5g06090 \\
\hline GPAT8 & & & NR & NR & -- & At4g00400 \\
\hline LPP1 & PA phosphatase & ER, PM? & NR & NR & NC & At2g01180 \\
\hline LPP2 & & & NR & NR & NC & At1g15080 \\
\hline LPP3 & & & NR & NR & $\mathrm{NC}$ & At3g02600 \\
\hline LPP\&1 & & IEM & NR & NR & $\mathrm{NC}$ & At3g50920 \\
\hline LPP\&2 & & & NM & NM & NM & At5g66450 \\
\hline $\mathrm{LPP} \gamma$ & & & NR & NR & NC & At5g03080 \\
\hline LPAT2 & Lyso-PA acyltransferase & ER & NR & NR & $\mathrm{NC}$ & At3g57650 \\
\hline LPAT3 & & & NR & NR & $\mathrm{NC}$ & At1g51260 \\
\hline LPAT4 & & & NR & NR & + & At1g75020 \\
\hline LPAT5 & & & NR & NR & - & At3g18850 \\
\hline MGD1 & MGDG synthase & IEM & NR & NR & ++ & At4g31780 \\
\hline MGD2 & & OEM & ++ & ++ & ++ & At5g20410 \\
\hline MGD3 & & OEM & ++ & ++ & ++ & At2g11810 \\
\hline NPC4 & Phospholipase C & PM & NM & NM & NM & At3g03530 \\
\hline NPC5 & & Cytosol & ++ & ++ & ++ & At3g03540 \\
\hline PAH1 & PA phosphohydrolase & ER & NR & NR & ++ & At3g09560 \\
\hline PAH2 & & & NR & NR & NC & At5g42870 \\
\hline PAK & PA kinase & PM, ER, & NR & NR & NC & At1g12430 \\
\hline PAP3 & PA phosphatase & Vacuole & -- & NR & -- & At1g14700 \\
\hline PGP1 & $\begin{array}{l}\text { Phosphatidylglycerolphosphate } \\
\text { synthase }\end{array}$ & IEM, Mitochondria & NR & NR & $\mathrm{NC}$ & At2g39290 \\
\hline PLD $\alpha 1$ & Phospholipase D & PM, Cytosol & NR & NR & $\mathrm{NC}$ & At3g15730 \\
\hline PLD $\delta$ & & & NR & NR & $\mathrm{NC}$ & At4g35790 \\
\hline PLD $\varepsilon$ & & & NR & NR & $\mathrm{NC}$ & At1g55180 \\
\hline PLD $\zeta 1$ & & Vesicles under PM & NR & NR & NC & At3g16785 \\
\hline PLDל̧2 & & Vacuole & ++ & ++ & ++ & At3g05630 \\
\hline SFR2 & $\begin{array}{l}\text { Galactolipid:galactolipid } \\
\text { galactosyl-transferase }\end{array}$ & OEM & NR & NR & $\mathrm{NC}$ & At3g06510 \\
\hline TGD1 & Lipid transport & Chloroplast envelope & NR & NR & $\mathrm{NC}$ & At1g19800 \\
\hline TGD2 & & & NR & NR & NC & At3g20320 \\
\hline TGD3 & & & NR & NR & $\mathrm{NC}$ & At1g65410 \\
\hline TGD4 & & ER & NR & NR & $\mathrm{NC}$ & At3g06960 \\
\hline
\end{tabular}

acyltransferases play a role in formation of DPG (cardiolipin), a specific mitochondrial lipid.

The ER glycerol-3-P and lyso-PA acyltransferases are involved in formation of most phospholipids: PC (phosphatidylcholine), PE (phosphatidylethanolamine), PI (phosphatidylinositol), PS (phosphatidylserine), and part of the cellular PG (phosphatidylgycerol) [14]. Furthermore, since about half of the galactolipids derive from ER-synthesized PC (referred to as eukaryotic galactolipids) these acyltransferases also display a role in formation of galactolipids. In fact a complex network of lipid synthetic pathways is issued from these acyltransferases and regulation between the different branches appears as essential. In Arabidopsis, LPAT2 encodes a ubiquitous ER-located lyso-PA-acyl CoA acyltransferase that is vital for the plant [15]. There are several other ER acyltransferases but all are not as important as LPAT2; for instance deletion of LPAT3 which is expressed in the male gametophyte does not display a lethal phenotype [15]. PA formed by ER acyltransferases can generate phospholipids via 2 different pathways: i) the CDP-DAG pathway and ii) the Kennedy pathway through dephosphorylation of PA into DAG. In yeast, both pathways generate PC and PE [16]. The CDP-DAG pathway is more active in the growth phase but nutrient depletion induces a shift towards the Kennedy pathway [16]. The Kennedy pathway indeed contributes to recycling the polar head of phospholipids such as choline and ethanolamine 
through phospholipases D (PLDs) and eventually to remodelling of membranes. The PA phosphatase Pah1p is critical for the shift from the CDP-DAG to the Kennedy pathway because it catalyzes the hydrolysis of PA into DAG, and furthermore because the Pah1pproduced DAG negatively regulates the level of expression of genes encoding the CDP-DAG pathway [16]. In plants, the biosynthetic pathways show similarity with yeast but also major differences partly related with galactolipid synthesis. The Kennedy pathway is preferentially used for formation of PC and PE and the CDP-DAG pathway for formation of PI, PG and probably PS (Fig. 1) [14]. Arabidopsis enzymes similar to the yeast Pah1p, AtPAH1 and AtPAH2, also control phospholipid synthesis but through a different system [17]. In the KO double mutant, pah1pah2, the expression of several other enzymes of the Kennedy pathway including the phosphoethanolamine N-methyltransferase PAEMT1 that catalyzes the first committed step of choline synthesis is enhanced as well as the rate of incorporation of choline into PC. These results indicate that activation of PAHs leads to inhibition of PC and PE syntheses. Interestingly, when plants are deprived of Pi, AtPAH1 and AtPAH2 activities are enhanced and AtPAHs contribute to the Pi restriction response by recycling of phospholipids into galactolipid $[17,18]$. However, it is likely important to distinguish two phases in the lipid response to Pi deprivation. In an early stage, a transient increase of PC precedes the increase of galactolipid synthesis [19] and several phospholipases D and C such as PLD $\zeta 1$, PLD $\zeta 2$, NPC4 and NPC5 are highly overexpressed $[20,21]$. Therefore, this suggests that, at this stage, the Kennedy pathway actively contributes to remodelling of phospholipids, notably of PE into PC [19]. This suggests that AtPAH1 and AtPAH2 are activated only after this stage, when galactolipid synthesis starts to increase. In this second stage, PLD $\zeta 1, P L D \zeta 2$ and NPC5 contribute to the recycling of DAG for galactolipid synthesis as was shown by mutant analysis [22-24]. The question is then how the eukaryotic DAG is imported into the chloroplast to reach the site of MGDG synthesis (Fig. 1). Several modes of transport have been proposed, mainly by relocation of PC, lyso-PC, DAG and/or PA (see [25]). A multimeric ABC transporter composed of three subunits, TGD1, TGD2 and TGD3, located to the chloroplast envelope, and probably associated with a fourth ER protein, TGD4, contributes to this import since TGD mutations strongly impair formation of the eukaryotic galactolipids [26]. Observation that the TGD2 subunit binds to PA [27] indicates that PA displays a role in the functioning of this complex but it is not yet clear how the complex works and whether PA is the only transported metabolite.

In chloroplasts, two different acyltransferases called ATS1 and ATS2 are involved in successive acylation of glycerol-3-P leading to formation of PA. ATS1 is specific for C18:1 chain at sn-1 position on glycerol-3-P [28] and ATS2, a chloroplast inner envelope protein, is specific for C16:0 chain [29-31]. Typical structure of PA is thus C18:1-sn-1, C16:0-sn-2, which is the characteristic prokaryotic structure found in chloroplast PG and in about half of the galactolipids (those are called prokaryotic galactolipids). PA is then either converted to CDP-diacylglycerol before incorporation into plastid PG, or it is converted to DAG by typical chloroplast envelope PA phosphatases before incorporation into galactolipids or sulfoquinovosyldiacylglycerol SQDG (Fig. 1). The vital importance of prokaryotic PA synthesis was indicated by invalidation of ATS2 gene, which gives an embryo development arrest at the stage of chloroplast formation [31,32]. In contrast, although a series of ats 1 mutants showed a strong impaired phenotype, the plants remained viable, probably due to a residual activity of ATS1 from RNAi invalidation, point mutations or partial sequence deletions [33]. Detailed lipid analysis from ats1 mutant indicated a complete loss of prokaryotic galactolipids and only a partial loss of chloroplast PG. This difference suggests that, when prokaryotic PA is supplied in limited amount, plastid PG synthesis is dominant over prokaryotic galactolipid synthesis, possibly because CDPDS has a higher affinity to PA than PA phosphatase. Like for the branching between CDPDAG and Kennedy pathways in the ER, PA phosphatase plays an essential role in the branching between PG and galactolipid synthesis in the envelope. A typical chloroplast envelope PA phosphatase has been described in the past [34]. This enzyme is tightly associated with the inner envelope membrane [35,36]. In contrast to most PA phosphatases, it shows an alkaline optimum $\mathrm{pH}$, a severe inhibition by $\mathrm{Mg}^{2+}$ suggesting a link with photosynthetic activity and a strong feedback inhibition by DAG suggesting that PA hydrolysis and galactolipid synthesis are coupled in the membrane. Three genes encoding chloroplast PA phosphatases have been identified in Arabidopsis: LPP $\gamma, \mathrm{LPP} \varepsilon 1$, and LPP\&2 [37]. Depletion of the LPP $\gamma$ led to a lethal mutant, whereas no phenotype was detected in the double knock out mutant lppe1lppe2. This suggests that either a specific pool of PA dephosphorylated by LPP $\gamma$ or a specific pool of DAG produced by LPP $\gamma$ plays a determinant role for the plant, indicating a need for analysis of substrate specificity of LPP $\gamma$.

Altogether, PA is an intermediate in numerous steps of galactolipid synthesis dispatched in the different membranes of the plant cell (Fig. 1). Although the lipid neosynthetic pathways are apparently redundant in several parts, it appears very frequently that, at each PA-involving step, deletion of several specific enzymes leads to a lethal phenotype. It is therefore likely that PA produced by these enzymes has specific properties that interfere with signalling processes. These properties should be related with fatty acid composition and localisation of PA. The transient localisation in a specific membrane spot and the activation of interaction capability with neighbouring components must be keys in the process.

\section{Is phosphatidic acid a central precursor for vital lipid synthesis in Apicomplexa, animal parasites with a relict plastid?}

Apicomplexa is a phylum of obligate intracellular parasites, including pathogens of medical importance such as the causative agent of malaria, Plasmodium spp. These unicellular eukaryotes harbour a non-photosynthetic plastid, the apicoplast, which is involved in vital metabolic pathways, one of them being lipid biosynthesis $[4,38,39]$. The demand in lipids required for parasite maintenance is met by combination of scavenging fatty acids from their host and de novo synthetic pathway [40]. PA is most likely to play a central part in the regulation between the different lipid fluxes. PA can be synthesized by the classical de novo two-steps acylation pathway within two compartments, the ER and the apicoplast. A glycerol-3-P acyltransferase, PfGPAT (PFL0620c) was characterized in Plasmodium falciparum ER, exhibiting a strong affinity for C16:0 and C16:1 [41]. Attempt to disrupt PfGPAT were unsuccessful suggesting an essential role of the protein. Regarding the apicoplast lipid synthesis pathway, it is well established that the prokaryotic type II fatty acid synthesis (FASII) is essential for Apicomplexa [42,43]. Homologues of chloroplast ATS1 and ATS2, PfATS1 and PfATS2, were predicted as apicoplast proteins in P. falciparum [39]. Furthermore, PfATS1 is a soluble apicoplast protein with an acyltransferase activity (C. Botté, personal communication) making the apicoplast a plausible site for PA neo-synthesis. However, nothing is known on the exact fate of the neosynthesized PA. The apicoplast lipid composition remains unknown but synthesis of galactolipids could be detected by metabolic labelling in Apicomplexa lysates [44,45]. Immunodetection using a polyclonal antibody raised against plant DGDG allowed to detect a digalactolipid-like epitope in Toxoplasma gondii (the Apicomplexa responsible for toxoplasmosis) and $P$. falciparum membranes and total lipid extracts $[45,46]$. Furthermore LC-MS/ 
MS approach showed the presence of two hexosyllipids in the membranes of $T$. gondii: hexosylceramides and hexosylglycerolipids, hexosylceramides being the most predominant class [46]. Together, these data are consistent with the potential synthesis of galactolipid classes in Apicomplexa by a pathway, which remains to be identified. Therefore prokaryotic PA could serve as a possible precursor for the synthesis of phospholipids and galactolipids. Due to the essentiality of the apicoplast for Apicomplexa and its plants origin, it would be important to investigate the apicoplast enzymes involved in the synthesis of PA for potential medical intervention.

\section{Mode of action of phosphatidic acid in signalling events}

PA is the simplest phospholipid but unlike other phospholipids, the phosphate head group of PA is a monoester and has a second pKa usually between 6.6 and 7.9 depending of the PA environment $[47,48]$. PA can thus carry one or two negative charges. Micromolar concentration of cations such as $\mathrm{Ca}^{2+}$ or $\mathrm{Mg}^{2+}$ at the bilayer surface can modify the charge of PA and the way it interacts with the membrane environment $[49,50]$. Since local concentration of $\mathrm{Ca}^{2+}$ and $\mathrm{Mg}^{2+}$ can fluctuate very rapidly in particular cellular condition, this chemical property of PA is an important feature to probe its environment. As a consequence PA can behave as a pH biosensor as notably reported in yeast for regulation of expression of phospholipid metabolic genes through PA-dependent sequestration in the ER of the transcription factor Opi1p [16,51,52]. Interestingly it has been recently demonstrated that during the first minutes of carbon deprivation the cytosolic $\mathrm{pH}$ of plant cells rises from 7.4 to stabilize at 7.8 and recovers to more acidic $\mathrm{pH}$ within $5 \mathrm{~min}$ after carbon repletion accompanying modification of level of soluble phosphorous metabolites [53]. The authors proposed that modification of cytosolic pH likely occurs also in response to Pi deprivation. Since enzymatic studies of galactolipid synthesizing enzymes showed different protonation levels of peripheral histidine residues interacting with cations in a $6-8 \mathrm{pH}$ range [54-56], one may question about the consequences on galactolipid synthesis.

The reduced size of its polar head confers to PA a cone shape, which can be amplified by desaturation of the fatty acyl chains. Because of this cone shape, accumulation of PA in a leaflet of a bilayer favours concave (negative) curvature of the membrane
[57]. The change in PA concentration can have central consequences on membrane trafficking. It has been shown in mammal chromaffin secretory cells that accumulation of PLD-produced PA at the inner leaflet of the plasma membrane causes a curvature which favours the fusion of the membrane with the contacting membrane of secretory vesicles $[58,59]$. The fusion was reproduced in PLD deficient cells by exogenous addition of lyso-PC at the outer leaflet of the plasma membrane. Among the twelve PLDs recorded in Arabidopsis, PLD 1 and PLD 22 are homologous to mammal PLDs and play a role in the formation of galactolipids induced by $\mathrm{Pi}$ deprivation [22,23]. Interestingly, PLDל2 has been shown to be also involved in root system architecture, auxin-dependent hypocotyl elongation and vesicle cycling [60,61]. PLD $\zeta 1$ has been localized in vesicles underlying the plasma membrane [62] and PLD $\zeta 2$ in the tonoplast [63], which suggests that they hydrolyze specific pools of phospholipids in the endomembrane system. At an early stage of Pi deprivation a transient increase of PLD $\zeta 2$-enriched domains has been observed in tonoplast [63]. These domains are close to mitochondria and chloroplasts whose contacts are themselves increased at this stage and concomitant with galactolipid transfer from chloroplasts to mitochondria [19,64]. On the model of what has been observed in mammal cells, one can propose that PLD 2 produced PA can facilitate hemifusion of membranes and eventually remodelling of lipids between tonoplast, chloroplasts and mitochondria.

PA can modify conformation of proteins leading to changes in the cell biology. Several target proteins have been identified in plants (Table 2). Some targets are protein kinases, protein phosphatases and $G$ proteins. They belong to signalling cascades involved in the abscissic acid signalling (i.e. ABI1 [65]), the ethylene signalling (i.e. CTR1 [66]), the salt stress response (i.e. MPK6 [67]) or the development of tissue (i.e. PDK1 [68]). Some others are related with the cytoskeleton such as the actin cap protein AtCP [69]. PA binding can induce a cascade of changes not only on membrane proteins but also in the surrounding domain inside the membrane [70]. Providing insight into the local chemistry and environments of functional groups within a molecule, FTIR difference spectrometry has been used to monitor the structural changes induced in a membrane by interaction of PA with a nicotinic acetylcholine receptor. This technique allowed to visualize increase in lateral

Table 2

Plant PA target proteins.

\begin{tabular}{|c|c|c|c|c|c|}
\hline Protein & Activity & Function & Effect of PA binding & PA binding region ${ }^{\mathrm{a}}$ & References \\
\hline ABI1 & Phosphatase & $\begin{array}{l}\text { Negative regulator of } \mathrm{ABA} \\
\text { signalling }\end{array}$ & $\begin{array}{l}\text { Membrane } \\
\text { recruitment/inhibition }\end{array}$ & ${ }^{60}$ GSHGSESRKVLISRINSPNLNMKESAAADIVVVDISAGD ${ }^{98}$ & [65] \\
\hline PP1 $c^{b}$ & Phosphatase & $\begin{array}{l}\text { Positive regulator of blue } \\
\text { light signalling }\end{array}$ & Inhibition & GEFDNAGAMMSVD-L-CSFQILPKA & [78] \\
\hline CDPK & Kinase & $\begin{array}{l}\text { Response to ROS in root hair } \\
\text { development and pathogens }\end{array}$ & Activation & ND & [68] \\
\hline CTR1 & Kinase & $\begin{array}{l}\text { Negative regulation } \\
\text { of ethylene response }\end{array}$ & Inhibition & $654-821$ & [66] \\
\hline MPK6 & Kinase & Salt stress response & Activation & ND & [67] \\
\hline PDK1 & Kinase & $\begin{array}{l}\text { Response to plant hormones, } \\
\text { regulation of root hair growth } \\
\text { and development }\end{array}$ & Inhibition & $391-491$ & {$[68]$} \\
\hline AGD7 & $\begin{array}{l}\text { Arf GTPase } \\
\text { Activating Protein }\end{array}$ & Vesicular trafficking & Activation & ND & [79] \\
\hline AtCP & Actin Capping Protein & Actin polymerization & Inhibition & ND & [69] \\
\hline MGD1 & MGDG synthase 1 & Galactolipids synthesis & Activation & ND & [2] \\
\hline PEAMT & $\begin{array}{l}\text { Phosphoethanolamine } \\
\text { N-Methyl-transferase }\end{array}$ & Phosphatidylcholine synthesis & Inhibition & ND & [80] \\
\hline PEPC & PEP Carboxylase & Oxaloacetate synthesis & Inhibition & ND & {$[81,82]$} \\
\hline TGD2 & ABC Transporter Subunit & Lipid import from ER to plastids & Binding & ${ }^{210}$ SVGPLHPECGKEGLIV ${ }^{225}$ & [27] \\
\hline
\end{tabular}

Abbreviation ND: Not determined.

a Phosphatidic acid binding region represents protein fragments that were found to be sufficient to bind PA. When available, amino acid sequence of the minimal PA binding region is indicated and essential amino acids are underlined.

${ }^{\mathrm{b}}$ Minimal PA binding domain of Vicia fabia PP1c was deduced from sequence homology with the PA binding domain of human PP1c $\gamma$. Only conserved residues are listed. 
packing of the lipid bilayer around the receptor and changes in the receptor conformation [50]. Conformational changes induced by PA can be associated with oligomer formation and recruitment of protein to membranes leading to enzyme activation (see for instance the work of [71,72] on the phospholipase PLC $\beta 1$ in mammals). Several works indicated in the past that PA and several anionic lipids such as PG can enhance MGDG synthase in plants (Fig. 1) [73-75]. We will hereafter analyze the specificity of its activation by PA.

\section{Regulation of MGDG synthase by phosphatidic acid}

MGDG synthase, MGD1, is essential for the formation of photosynthetic membranes [76]. Regulation of MGD1 by PA and PG was recently analyzed on native enzyme in leaf extracts and on the recombinant At $\Delta$ 1-137 MGD1 enzyme [2]. No activity was measured when the enzyme was totally depleted of lipids except for the supply of its own substrate DAG. However addition of a very low concentration of PA was sufficient to promote the activity. Enzyme activation was also possible with PG but required much higher concentrations. Activation constants were $0.2 \mathrm{~mol} \%$ for PA and $5 \mathrm{~mol} \%$ for PG. No activity was recorded with PA or PG in absence of DAG. These results therefore suggested an allosteric regulation of MGDG synthase by PA and PG. Binding of PA and PG on MGD1 was indeed verified by lipid/protein overlay assay. PG concentration in the envelope is close to $8 \mathrm{~mol} \%$ and PA is not detected in the envelope. However activity of the native MGDG synthase in the chloroplast envelope lipid background was drastically reduced after the envelope was incubated in optimum condition for PA phosphatase, the lost activity being restored by resupplying PA. $\mathrm{K}_{0.5}$ of the spinach chloroplast envelope MGDG synthase for PA was estimated to $0.6 \mathrm{~mol} \%$. This experiment indicated the crucial importance of a very low level of PA in the chloroplast envelope for MGD1 activity. Several molecular species of PA were able to activate the enzyme, some eukaryotic as well as prokaryotic molecular species, suggesting a possible regulation by different sources of cellular PA, from chloroplast and nonchloroplast origins. On the other hand, PG appeared also important for MGDG synthase activity since PA and PG did not activate the enzyme by the same process. The curve of enzyme velocity versus lipid activator concentration looked sigmoid with PA but hyperbolic with PG, indicative of a simpler way of interaction of the enzyme with PG than with PA. Although a higher concentration of PG was required to reach apparent maximum velocity, PG activation allowed a higher apparent maximum velocity than PA activation, closer to the true maximum velocity of the enzyme. Different and complementary activations were confirmed by observation of the synergic effects of PA and PG on the enzyme. Analysis of salt effects indicated hydrophobic interaction of PA with the enzyme, i.e. increased activation by PA with high salt concentration, and electrostatic interaction of PG, i.e. decreased activation by PG with high salt concentration, and lastly, point mutation of the enzyme allowed determination of 2 amino acids specifically important for activation by PG [2]. It is now important to understand the synergic effect of PA and PG on the MGDG synthase activity in vivo.

In leaves, PLDY2 is a source of PA that can activate MGDG synthase since MGDG synthase activity is weaker in the pldל2 knock out mutant unless an exogenous source of PA is added [2]. With regards to the role of PLD 22 under the initial stage of Pi deprivation and because PLD 22 is located on the membrane of vacuole where Pi is stored (Fig. 1) [20,63], it has been proposed that PA produced by PLDY2 can tune MGDG synthesis as a function of Pi availability. However, because of different roles of PA in multiple cellular functions, activation of MGD1 by PA suggests many different possibilities of control of chloroplast biogenesis through MGDG synthesis. Import of non-chloroplast PA and balance between PG synthesis and galactolipid synthesis in the chloroplast envelope are points that will be interesting to study in the future.

\section{Perspectives}

In plants, PA is a central precursor in both phospholipid and galactolipid synthesis. Mutant analysis indicated that some specific enzymes involved in de novo PA synthesis such as several lyso-PA acyltransferases (for instance LPAT2 and ATS2) are vital for the plant. Some PA phosphatases (for instance PAH1, PAH2 and LPP $\gamma$ ) look also essential. It will be interesting to analyze what makes these enzymes essential. This must be related with control of expression of the proteins and regulation of enzyme activity and ultimately with modification of PA level. Two important points to consider will be the exact membrane location of these enzymes and the lipid substrates they can be supplied with, at this location. Another important point will be to characterize how modification of level of specific molecular species of PA can affect key target proteins such as, MGD1, the essential MGDG synthase in formation of photosynthetic membranes. Molecular tools to follow quantity and location of different molecular species of PA in the cell would add precious information to unravel the way PA acts as a signal in the plant cell.

\section{Acknowledgements}

Our research is supported by Centre National de la Recherche Scientifique (CNRS), Commissariat à l'Energie Atomique et aux Energies Alternatives (CEA), Institut National de la Recherche Agronomique (INRA) and Université Joseph Fourier (UJF). CB is supported by a Marie-Curie FP7 fellowship from the European Union; YY is supported by a grant from the Tokyo Institute of Technology, Japan; EM and MB are supported by a blue sky grant from Agence Nationale de la Recherche, France. We thank Hiroyuki Ohta for his help in finding a financial support to YY.

\section{References}

[1] K. Awai, E. Maréchal, M.A. Block, D. Brun, T. Masuda, H. Shimada, K. Takamiya, H. Ohta, J. Joyard, Two types of MGDG synthase genes, found widely in both 16:3 and 18:3 p.ants, differentially mediate galactolipid syntheses in photosynthetic and nonphotosynthetic tissues in Arabidopsis thaliana, Proc. Natl. Acad Sci. USA 98 (2001) 10960-10965.

[2] E. Dubots, M. Audry, Y. Yamaryo, O. Bastien, H. Ohta, C. Breton, E. Maréchal, M.A. Block, Activation of the chloroplast monogalactosyldiacylglycerol synthase MGD1 by phosphatidic acid and phosphatidylglycerol, J. Biol. Chem. 285 (2010) 6003-6011.

[3] N. Sato, M. Hagio, H. Wada, M. Tsuzuki, Requirement of phosphatidylglycerol for photosynthetic function in thylakoid membranes, Proc. Natl. Acad. Sci. U S A 97 (2000) 10655-10660.

[4] G.I. McFadden, M.E. Reith, J. Munholland, N. Lang-Unnasch, Plastid in human parasites, Nature 381 (1996) 482.

[5] S. Kohler, C.F. Delwiche, P.W. Denny, L.G. Tilney, P. Webster, R.J. Wilson, J.D. Palmer, D.S. Roos, A plastid of probable green algal origin in Apicomplexan parasites, Science 275 (1997) 1485-1489.

[6] E. Marechal, M.F. Cesbron-Delauw, The apicoplast: a new member of the plastid family, Trends Plant Sci. 6 (2001) 200-205.

[7] X. Wang, S.P. Devaiah, W. Zhang, R. Welti, Signaling functions of phosphatidic acid, Prog. Lipid Res. 45 (2006) 250-278.

[8] R. Welti, W. Li, M. Li, Y. Sang, H. Biesiada, H.E. Zhou, C.B. Rajashekar, T.D. Williams, X. Wang, Profiling membrane lipids in plant stress responses. Role of phospholipase D alpha in freezing-induced lipid changes in Arabidopsis, J. Biol. Chem. 277 (2002) 31994-32002.

[9] B.O. Bargmann, A.M. Laxalt, B. ter Riet, B. van Schooten, E. Merquiol, C. Testerink, M.A. Haring, D. Bartels, T. Munnik, Multiple PLDs required for high salinity and water deficit tolerance in plants, Plant Cell Physiol. 50 (2009) 78-89.

[10] W. Li, M. Li, W. Zhang, R. Welti, X. Wang, The plasma membrane-bound phospholipase D delta enhances freezing tolerance in Arabidopsis thaliana, Nat. Biotechnol. 22 (2004) 427-433.

[11] M. Uemura, R.A. Joseph, P.L. Steponkus, Cold acclimation of Arabidopsis thaliana (Effect on plasma membrane lipid composition and freeze-induced lesions), Plant Physiol. 109 (1995) 15-30. 
[12] M. Fritz, H. Lokstein, D. Hackenberg, R. Welti, M. Roth, U. Zahringer, M. Fulda W. Hellmeyer, C. Ott, F.P. Wolter, E. Heinz, Channeling of eukaryotic diac ylglycerol into the biosynthesis of plastidial phosphatidylglycerol, J. Biol. Chem. 282 (2007) 4613-4625.

[13] M. Frentzen, M. Neuburger, J. Joyard, R. Douce, Intraorganelle localization and substrate specificities of the mitochondrial acyl-CoA: sn-glycerol-3-phosphate O-acyltransferase and acyl-CoA: 1-acyl-sn-glycerol-3-phosphate O-acyltransferase from potato tubers and pea leaves, Eur. J. Biochem. 187 (1990) 395-402.

[14] J. Ohlrogge, J. Browse, Lipid biosynthesis, Plant Cell 7 (1995) 957-970.

[15] H.U. Kim, Y. Li, A.H. Huang, Ubiquitous and endoplasmic reticulum-located lysophosphatidyl acyltransferase, LPAT2, is essential for female but no male gametophyte development in Arabidopsis, Plant Cell 17 (2005) 1073-1089.

[16] G.M. Carman, S.A. Henry, Phosphatidic acid plays a central role in the transcriptional regulation of glycerophospholipid synthesis in Saccharomyces cerevisiae, J. Biol. Chem. 282 (2007) 37293-37297.

[17] P.J. Eastmond, A.L. Quettier, J.T. Kroon, C. Craddock, N. Adams, A.R. Slabas, Phosphatidic acid phosphohydrolase 1 and 2 regulate phospholipid synthesis at the endoplasmic reticulum in Arabidopsis, Plant Cell 22 (2010) 2796-2811.

[18] Y. Nakamura, R. Koizumi, G. Shui, M. Shimojima, M.R. Wenk, T. Ito, H. Ohta, Arabidopsis lipins mediate eukaryotic pathway of lipid metabolism and cope critically with phosphate starvation, Proc. Natl. Acad. Sci. USA 106 (2009) 20978-20983.

[19] J. Jouhet, E. Marechal, R. Bligny, J. Joyard, M.A. Block, Transient increase of phosphatidylcholine in plant cells in response to phosphate deprivation, FEBS Lett. 544 (2003) 63-68.

[20] J. Misson, K.G. Raghothama, A. Jain, J. Jouhet, M.A. Block, R. Bligny, P. Ortet, A. Creff, S. Somerville, N. Rolland, P. Doumas, P. Nacry, L. Herrerra-Estrella, L. Nussaume, M.C. Thibaud, A genome-wide transcriptional analysis using Arabidopsis thaliana Affymetrix gene chips determined plant responses to phosphate deprivation, Proc. Natl. Acad. Sci. U S A 102 (2005) 11934-11939.

[21] R. Morcuende, R. Bari, Y. Gibon, W. Zheng, B.D. Pant, O. Blasing, B. Usadel, T. Czechowski, M.K. Udvardi, M. Stitt, W.R. Scheible, Genome-wide reprogramming of metabolism and regulatory networks of Arabidopsis in response to phosphorus, Plant Cell Environ. 30 (2007) 85-112.

[22] A. Cruz-Ramirez, A. Oropeza-Aburto, F. Razo-Hernandez, E. Ramirez-Chavez L. Herrera-Estrella, Phospholipase DZ2 p.ays an important role in extraplastidic galactolipid biosynthesis and phosphate recycling in Arabidopsis roots, Proc. Natl. Acad. Sci. U S A 103 (2006) 6765-6770.

[23] M.Y. Li, R. Welti, X.M. Wang Quantitative profiling of i polar glycerolipids in response to phosphorus starvation. Roles of Phospholipases D zeta 1 and D zeta 2 in phosphatidylcholine hydrolysis and digalactosyldiacylglycerol accumulation in phosphorus-starved plants, Plant Physiol. 142 (2006) 750-761.

[24] N. Gaude, Y. Nakamura, W.R. Scheible, H. Ohta, P. Dormann, Phospholipase C5 (NPC5) is involved in galactolipid accumulation during phosphate limitation in leaves of Arabidopsis, Plant J. 56 (2008) 28-39.

[25] J. Jouhet, E. Marechal, M.A. Block, Glycerolipid transfer for the building of membranes in plant cells, Prog. Lipid Res. 46 (2007) 37-55.

[26] C. Benning, Mechanisms of lipid transport involved in organelle biogenesis in plant cells, Annu. Rev. Cell Dev. Biol. 25 (2009) 71-91.

[27] B. Lu, C. Benning, A 25-amino acid sequence of the Arabidopsis TGD2 p.otein is sufficient for specific binding of phosphatidic acid, J. Biol. Chem. 284 (2009) $17420-17427$.

[28] I. Nishida, Y. Tasaka, H. Shiraishi, N. Murata, The gene and the RNA for the precursor to the plastid-located glycerol-3-phosphate acyltransferase of Arabidopsis thaliana, Plant Mol. Biol. 21 (1993) 267-277.

[29] M. Frentzen, E. Heinz, T.A. McKeon, P.K. Stumpf, Specificities and selectivities of glycerol-3-phosphate acyltransferase and monoacylglycerol-3-phosphate acyltransferase from pea and spinach chloroplasts, Eur. J. Biochem. 129 (1983) 629-636

[30] M. Ferro, D. Salvi, S. Brugiere, S. Miras, S. Kowalski, M. Louwagie, J. Garin J. Joyard, N. Rolland, Proteomics of the chloroplast envelope membranes from Arabidopsis thaliana, Mol. Cell Proteomics 2 (2003) 325-345.

[31] B. Yu, S. Wakao, J. Fan, C. Benning, Loss of plastidic lysophosphatidic acid acyltransferase causes embryo-lethality in Arabidopsis, Plant Cell Physiol. 45 (2004) 503-510.

[32] H.U. Kim, A.H. Huang, Plastid lysophosphatidyl acyltransferase is essential for embryo development in Arabidopsis, Plant Physiol. 134 (2004) 1206-1216.

[33] C. Xu, B. Yu, A.J. Cornish, J.E. Froehlich, C. Benning, Phosphatidylglycerol biosynthesis in chloroplasts of Arabidopsis mutants deficient in acyl-ACP glycerol-3-phosphate acyltransferase, Plant J. 47 (2006) 296-309.

[34] J. Joyard, R. Douce, Characterization of phosphatidate phosphohydrolase activity associated with chloroplast envelope membranes, FEBS Lett. 102 (1979) 147-150

[35] M.A. Block, A.-J. Dorne, J. Joyard, R. Douce, The phosphatidic acid phosphatase of the chloroplast envelope is located on the inner envelope membrane, FEBS Lett. 164 (1983) 111-115.

[36] A. Malherbe, M.A. Block, J. Joyard, R. Douce, Feedback inhibition of phosphatidate phosphatase from spinach chloroplast envelope membranes by diacylglycerol, J. Biol. Chem. 267 (1992) 23546-23553.

[37] Y. Nakamura, M. Tsuchiya, H. Ohta, Plastidic phosphatidic acid phosphatases identified in a distinct subfamily of lipid phosphate phosphatases with prokaryotic origin, J. Biol. Chem. 282 (2007) 29013-29021.
[38] M.E. Fichera, D.S. Roos, A plastid organelle as a drug target in apicomplexan parasites, Nature 390 (1997) 407-409.

[39] S.A. Ralph, G.G van Dooren, R.F. Waller, M.J. Crawford, M.J. Fraunholz, B.J. Foth, C.J. Tonkin, D.S. Roos, G.I. McFadden, Tropical infectious diseases: metabolic maps and functions of the Plasmodium falciparum apicoplast, Nat. Rev. Microbiol. 2 (2004) 203-216.

[40] A.S. Tarun, A.M. Vaughan, S.H. Kappe, Redefining the role of de novo fatty acid synthesis in Plasmodium parasites, Trends Parasitol. 25 (2009) 545-550.

[41] T.C. Santiago, R. Zufferey, R.S. Mehra, R.A. Coleman, C.B. Mamoun, The Plasmodium falciparum PfGatp is an endoplasmic reticulum membrane protein important for the initial step of malarial glycerolipid synthesis, J. Biol. Chem. 279 (2004) 9222-9232.

[42] R.F. Waller, P.J. Keeling R.G. Donald, B. Striepen, E. Handman, N. LangUnnasch, A.F. Cowman, G.S. Besra, D.S. Roos, G.I. McFadden, Nuclear-encoded proteins target to the plastid in Toxoplasma gondii and Plasmodium falciparum, Proc. Natl. Acad. Sci. U S A 95 (1998) 12352-12357.

[43] A.M. Vaughan, M.T. O'Neill, A.S. Tarun, N. Camargo, T.M. Phuong, A.S. Aly, A.F. Cowman, S.H. Kappe, Type II fatty acid synthesis is essential only for malaria parasite late liver stage development, Cell Microbiol. 11 (2009) 506-520.

[44] E. Maréchal, N. Azzouz, C.S. de Macedo, M.A. Block, J.E. Feagin, R.T. Schwarz J. Joyard, Synthesis of chloroplast galactolipids in apicomplexan parasites, Eukaryot. Cell 1 (2002) 653-656.

[45] C. Bisanz, O. Bastien, D. Grando, J. Jouhet, E. Marechal, M.F. Cesbron Delauw, Toxoplasma gondii acyl-lipid metabolism: de novo synthesis from apicoplastgenerated fatty acids versus scavenging of host cell precursors, Biochem. J. 394 (2006) 197-205.

[46] C. Botté, N. Saidani, R. Mondragon, M. Mondragon, G. Isaac, E. Mui, R. McLeod, J.F. Dubremetz, H. Vial, R. Welti, M.F. Cesbron Delauw, C. Mercier, E. Maréchal, Subcellular localization and dynamics of a digalactolipid-like epitope in Toxoplasma gondii, J. Lipid Res. 49 (2008) 746-762.

[47] A. Bhushan, M.G. McNamee, Correlation of phospholipid structure with functional effects on the nicotinic acetylcholine receptor. A modulatory role for phosphatidic acid, Biophys. J. 64 (1993) 716-723.

[48] E.E. Kooijman, K.M. Carter, E.G. van Laar, V. Chupin, K.N. Burger, B. de Kruijff, What makes the bioactive lipids phosphatidic acid and lysophosphatidic acid so special? Biochemistry 44 (2005) 17007-17015.

[49] J. Faraudo, A. Travesset, Phosphatidic acid domains in membranes: effect of divalent counterions, Biophys. J. 92 (2007) 2806-2818.

[50] R.M. Sturgeon, J.E. Baenziger, Cations mediate interactions between the nicotinic acetylcholine receptor and anionic lipids, Biophys. J. 98 (2009) 989-998.

[51] C.J. Loewen, M.L. Gaspar, S.A. Jesch, C. Delon, N.T. Ktistakis, S.A. Henry, T.P. Levine, Phospholipid metabolism regulated by a transcription factor sensing phosphatidic acid, Science 304 (2004) 1644-1647.

[52] B.P. Young, J.J. Shin, R. Orij, J.T. Chao, S.C. Li, X.L. Guan, A. Khong, E. Jan, M.R. Wenk, W.A. Prinz, G.J. Smits, C.J. Loewen, Phosphatidic acid is a pH biosensor that links membrane biogenesis to metabolism, Science 329 (2010) 1085-1088.

[53] E. Gout, R. Bligny, R. Douce, A.M. Boisson, C. Rivasseau, Early response of plant cell to carbon deprivation: in vivo 31P-NMR spectroscopy shows a quasiinstantaneous disruption on cytosolic sugars, phosphorylated intermediates of energy metabolism, phosphate partitioning, and intracellular pHs, New Phytol. 189 (2010) 135-147.

[54] E. Maréchal, C. Miège, M.A. Block, R. Douce, J. Joyard, The catalytic site of monogalactosyldiacylglycerol synthase from spinach chloroplast envelope membranes. Biochemical analysis of the structure and of the metal content, J. Biol. Chem. 270 (1995) 5714-5722.

[55] C. Miège, E. Maréchal, M. Shimojima, K. Awai, M.A. Block, H. Ohta, K. Takamiya, R. Douce, J. Joyard, Biochemical and topological properties of type A MGDG synthase, a spinach chloroplast envelope enzyme catalyzing the synthesis of both prokaryotic and eukaryotic MGDG, Eur. J. Biochem. 265 (1999) 990-1001.

[56] Y. Nishiyama, H. Hardre-Lienard, S. Miras, C. Miege, M.A. Block, F. Revah, J. Joyard, E. Marechal, Refolding from denatured inclusion bodies, purification to homogeneity and simplified assay of MGDG synthases from land plants, Protein Expr. Purif. 31 (2003) 79-87.

[57] E.E. Kooijman, V. Chupin, B. de Kruijff, K.N. Burger, Modulation of membrane curvature by phosphatidic acid and lysophosphatidic acid, Traffic 4 (2003) $162-174$.

[58] A. Begle, P. Tryoen-Toth, J. de Barry, M.F. Bader, N. Vitale, ARF6 regulates the synthesis of fusogenic lipids for calcium-regulated exocytosis in neuroendocrine cells, J. Biol. Chem. 284 (2009) 4836-4845.

[59] M. Zeniou-Meyer, A. Begle, M.F. Bader, N. Vitale, The Coffin-Lowry syndromeassociated protein RSK2 controls neuroendocrine secretion through the regulation of phospholipase D1 at the exocytotic sites, Ann. N. Y. Acad. Sci. 1152 (2009) 201-208.

[60] G. Li, H.W. Xue, Arabidopsis PLDzeta2 regulates vesicle trafficking and is required for auxin response, Plant Cell 19 (2007) 281-295.

[61] M. Li, C. Qin, R. Welti, X. Wang, Double knockouts of phospholipases Dz1 and $\mathrm{Dz2}$ in Arabidopsis affect root elongation during phosphate-limited growth but do not affect root hair patterning, Plant Physiol. 140 (2006) 761-770.

[62] Y. Ohashi, A. Oka, R. Rodrigues-Pousada, M. Possenti, I. Ruberti, G. Morelli, T. Aoyama, Modulation of phospholipid signaling by GLABRA2 in root-hair pattern formation, Science 300 (2003) 1427-1430. 
[63] Y. Yamaryo, E. Dubots, C. Albrieux, B. Baldan, M.A. Block, Phosphate availability affects the tonoplast localization of PLDzeta2, an Arabidopsis thaliana phospholipase D, FEBS Lett. 582 (2008) 685-690.

[64] J. Jouhet, E. Maréchal, B. Baldan, R. Bligny, J. Joyard, M.A. Block, Phosphate deprivation induces transfer of DGDG galactolipid from chloroplast to mitochondria, J. Cell Biol. 167 (2004) 863-874.

[65] W. Zhang, C. Qin, J. Zhao, X. Wang, Phospholipase D alpha 1-derived phosphatidic acid interacts with $\mathrm{ABI} 1$ p.osphatase $2 \mathrm{C}$ and regulates abscisic acid signaling, Proc. Natl. Acad. Sci. U S A 101 (2004) 9508-9513.

[66] C. Testerink, P.B. Larsen, D. van der Does, J.A. van Himbergen, T. Munnik, Phosphatidic acid binds to and inhibits the activity of Arabidopsis CTR1, J. Exp. Bot. 58 (2007) 3905-3914.

[67] L. Yu, J. Nie, C. Cao, Y. Jin, M. Yan, F. Wang, J. Liu, Y. Xiao, Y. Liang, W. Zhang, Phosphatidic acid mediates salt stress response by regulation of MPK6 in Arabidopsis thaliana, New Phytol. 188 (2010) 762-773.

[68] R.G. Anthony, R. Henriques, A. Helfer, T. Meszaros, G. Rios, C. Testerink, T. Munnik, M. Deak, C. Koncz, L. Bogre, A protein kinase target of a PDK1 signalling pathway is involved in root hair growth in Arabidopsis, EMBO J. 23 (2004) 572-581.

[69] S. Huang, L. Gao, L. Blanchoin, C.J. Staiger, Heterodimeric capping protein from Arabidopsis is regulated by phosphatidic acid, Mol. Biol. Cell 17 (2006) 1946-1958.

[70] E.E. Kooijman, D.P. Tieleman, C. Testerink, T. Munnik, D.T. Rijkers, K.N. Burger B. de Kruijff, An electrostatic/hydrogen bond switch as the basis for the specific interaction of phosphatidic acid with proteins, J. Biol. Chem. 282 (2007) 11356-11364.

[71] I. Litosch, R. Pujari, S.J. Lee, Phosphatidic acid regulates signal output by G protein coupled receptors through direct interaction with phospholipase C-beta(1), Cell Signal. 21 (2009) 1379-1384.

[72] E.M. Ross, D. Mateu, A.V. Gomes, C. Arana, T. Tran, I. Litosch, Structural determinants for phosphatidic acid regulation of phospholipase C-beta1, J. Biol. Chem. 281 (2006) 33087-33094.

[73] J. Coves, J. Joyard, R. Douce, Lipid requirement and kinetic studies of solubilized UDP-galactose:diacylglycerol galactosyltransferase activity from spinach chloroplast envelope membranes, Proc. Natl. Acad. Sci. U S A 85 (1988) 4966-4970

[74] A.A. Kelly, A. Öhman, A. Sedoud, A. Wieslander, Membrane homeostasis mechanisms for plant glycosyl-transferases involved in galactolipid biosynthesis: biochemical and in-silico structure studies of key enzymes. in:
C. Benning, J. Olhlrogge (Eds.), Current Advances in the Biochemistry and Cell Biology of Plant Lipids. Aardvark Global Publishing Company, Salt Lake City, 2007, pp. 25-31.

[75] H. Ohta, M. Shimojima, T. Arai, T. Masuda, Y. Shioi, K. Takamiya, UDP-galactose:diacylglycerol galactosyltransferase in cucumber seedlings:purification of the enzyme and the activation by phosphatidic acid. in: J.C. Kader, P. Mazliak (Eds.), Plant Lipid Metabolism. Kluwer Academic Publishers, Dordrecht, 1995, pp. 152-155.

[76] K. Kobayashi, M. Kondo, H. Fukuda, M. Nishimura, H. Ohta, Galactolipid synthesis in chloroplast inner envelope is essential for proper thylakoid biogenesis, photosynthesis, and embryogenesis, Proc. Natl. Acad. Sci. U S A 104 (2007) 17216-17221.

[77] J. Joyard, M. Ferro, C. Masselon, D. Seigneurin-Berny, D. Salvi, J. Garin, N. Rolland, Chloroplast proteomics highlights the subcellular compartmentation of lipid metabolism, Prog. Lipid Res. 49 (2010) 128-158.

[78] A. Takemiya, K. Shimazaki, Phosphatidic acid inhibits blue light-induced stomatal opening via inhibition of protein phosphatase 1 [corrected], Plant Physiol. 153 (2010) 1555-1562.

[79] M.K. Min, S.J. Kim, Y. Miao, J. Shin, L. Jiang, I. Hwang, Overexpression of Arabidopsis AGD7 causes relocation of Golgi-localized proteins to the endoplasmic reticulum and inhibits protein trafficking in plant cells, Plant Physiol. 143 (2007) 1601-1614.

[80] R. Jost, O. Berkowitz, J. Shaw, J. Masle, Biochemical characterization of two wheat phosphoethanolamine N-methyltransferase isoforms with differen sensitivities to inhibition by phosphatidic acid, J. Biol. Chem. 284 (2009) 31962-31971.

[81] J.A. Monreal, F. McLoughlin, C. Echevarria, S. Garcia-Maurino, C. Testerink, Phosphoenolpyruvate carboxylase from C4 leaves is selectively targeted for inhibition by anionic phospholipids, Plant Physiol. 152 (2010) 634-638.

[82] C. Testerink, H.L. Dekker, Z.Y. Lim, M.K. Johns, A.B. Holmes, C.G. Koster, N.T. Ktistakis, T. Munnik, Isolation and identification of phosphatidic acid targets from plants, Plant J. 39 (2004) 527-536.

[83] P.D. Bates, T.P. Durrett, J.B. Ohlrogge, M. Pollard, Analysis of acyl fluxes through multiple pathways of triacylglycerol synthesis in developing soybean embryos, Plant Physiol. 150 (2009) 55-72.

[84] S.C. Lung, R.J. Weselake, Diacylglycerol acyltransferase: a key mediator of plant triacylglycerol synthesis, Lipids 41 (2006) 1073-1088.

[85] T. Munnik, C. Testerink, Plant phospholipid signaling: "in a nutshell”, J. Lipid Res. 50 (Suppl) (2009) S260-265. 